\title{
Latencies on last error in paired-associate learning'
}

\author{
Madeleine Schlag-Rey, UNIVERSITY OF CALIFORNIA, LOS ANGELAS \\ Guy Groen and Patriek Suppes, STANFORD UNIVERITY
}

\begin{abstract}
Response latency was measured in a paired-associate experiment with. 3 response alternatives and 12 stimulus items. The mean error latency showed a rapid initial increase compared to the mean success latency. Further analysis revealed that the mean latency on the trial of last error was considerably larger than mean latencies on trials before or after the trial of last error.

\section{Introduction}

This paper reports some results of a paired-associate learning experiment in which the response latency was measured on each trial. At the time it was performed (1962) almost no empirical information exsisted as to the trial-by-trial variation of response latency in pairedassociate learning.

Recently, Millward (1964) has reported an experiment that resembles the present one in many respects. The main difference is that Millward used digits as stimuli and two response alternatives whereas the present experiment used nonsense syllables and three response alternatives.

This report will confine itself to those results that differ from Millward's. A more detailed presentation of the results, together with a theoretical analysis, will be published in the near future.

\section{Merhod}

On each trial, one of a set of 12 stimuli was presented to $S$. $S$ responded by pressing one of three keys. A reinforcing light was placed above each key. Upon S's response, one of the lights lit up indicating the correct response.

The stimulus items were drawn from a list of nonsense syllables of low association value (Hilgard, 1951, Table 9). They were presented in an illuminated screen mounted in the center of a vertical black panel. The response keys were placed $1 \mathrm{~cm}$ apart and could easily be reached by a single finger without moving the hand.

A buzzer indicated the start of the trial, shortly preceding the stimulus presentaion. After S's response, the stimulus and the reinforcing light both stayed on for 2 sec. The next trial started after a delay of $2.5 \mathrm{sec}$. The presentation of all experimenterdetermined events was automatically controlled. The response latency, from the onset of the stimulus to the occurrence of the response, was measured by a Beckman Universal Eput and Timer $5230 \mathrm{P}$.

Forty college students were used as subjects. They were not aware that their response latencies were being measured. Each $S$ was run individually for two sessions, about a week apart. Session 1 was identical to Session 2 except that a new set of stimulus items was used.

Each $\mathrm{S}$ was run for 300 trials on each session, following nine practice trials. Each stimulus item was presented 25 times, once in each block of 12 trials. The order of presentation was randomized within blocks. Each response was randomly assigned to four stimulus items. The assignments of response to key and subject to condition were also randomized.

\section{Hesults}

In the analysis of the data, each subject-item was treated as a separate protocol. A criterion of five trials to perfect learning was used, and all subject-items which had not reached criterion by trial 25 were eliminated from the analysis. On Session 1, 468 subject-items,

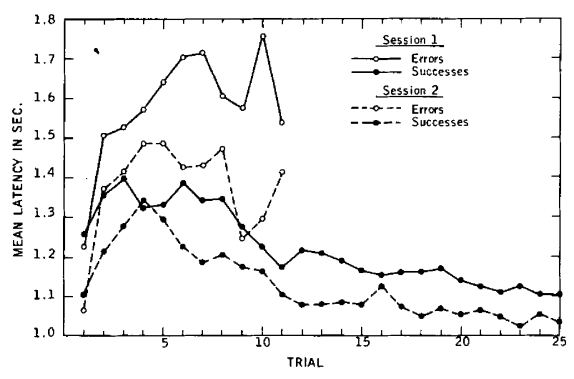

Fig. 1. Mean latency curves conditionalized on errors and successes.

and on Session 2, 470 subject-items, out of a possible 480 , met this criterion. Post-criterion errors were treated as successes. Each session was analysed separately. The results of the two sessions were similar except that learning occurred sooner and latencies tended to be shorter on Session 2 .

Figure 1 shows the mean latency of successes and errors as a function of the trials. Error latencies after trial 11 are not included because they are based on less than $10 \%$ of the data. It should be noted that the mean error latency rises sharply and is considerably greater than the mean success latency.

A possibility exists that the sharp increase in the mean error latency was due to subjects taking a long time to respond to items that they found difficult. Also, the curves of Fig. 1 do not clearly distinguish between latencies on pre-criterion trials and latencies on post-criterion trials. As a result, a further analysis was performed in which all protocols with the same trial of last error were grouped together and averages computed for each group separately. Mean error latencies, mean success latencies and mean overall latencies were computed in this fashion. The result of this analysis for the mean overall latencies is partially shown in Table 1. In this table, group $i$ denotes the group of protocols with last error on trial i. Group 0 is the set of protocols with no pre-criterion errors. For group $k$, the distance of trial $n$ from the trial of last error is equal to $n-k$. Thus, the entry in row $i$ and column $j$ is the mean latency on trial $i+j$ averaged over all protocols with last error on trial i.

Neither Table 1 nor similar analyses of success and error latencies reveal any evidence for a consistent increase in latency as a function of item difficulty that would account for the sharp initial increase in the mean error latency curve. However, Table 1 indicates an alternative explanation. If the entries in column 0 are compared with the entries in column -1 and column 1 , it is apparent that the latency rises to a maximum on the 
Table 1. Mean overall latencies for Session 1 as a function of item difficulty and distance from trial of last error.

DISTANCE FROM TRIAL OF LAST ERROR

\begin{tabular}{|c|c|c|c|c|c|c|c|c|c|c|c|c|c|c|c|c|c|}
\hline Group & $\begin{array}{l}\text { Number } \\
\text { of } \\
\text { Protocols }\end{array}$ & -8 & -7 & -6 & -5 & -4 & -3 & -2 & -1 & 0 & 1 & 2 & 3 & 4 & 5 & 6 & 7 \\
\hline 0 & 37 & & & & & & & & & & 1.21 & $1 \cdot 36$ & 1.29 & 1.14 & 1.30 & 1.22 & 1.20 \\
\hline 1 & 48 & & & & & & & & & 1.09 & 1.13 & 1.18 & 1.07 & 1.14 & 2.15 & 1.01 & 1.11 \\
\hline 2 & 35 & & & & & & & & 1.16 & 1.58 & 1.37 & 1.34 & 1.09 & 1.13 & 1.22 & 1.06 & 1.08 \\
\hline 3 & 29 & & & & & & & 1.28 & 1.55 & 1.85 & 1.40 & 1.41 & 1.50 & 1.49 & 1.19 & 1.26 & 1.11 \\
\hline 4 & 44 & & & & & & 1.16 & 1.39 & 1.39 & 1.61 & 1.27 & 1.37 & 1.22 & 1.21 & 1.11 & 1.12 & 1.12 \\
\hline 5 & 50 & & & & & 1.23 & 1.51 & 1.52 & 1.43 & 1.73 & 1.49 & 1.46 & 1.45 & 1.36 & 1.22 & 1.17 & 1.30 \\
\hline 6 & 31 & & & & 1.23 & 1.67 & 1.53 & 1.51 & 1.65 & 1.76 & 1.41 & 1.49 & 1.39 & 1.20 & $1 .=7$ & 1.35 & 1.16 \\
\hline 7 & 28 & & & 1.19 & 1.44 & 1.55 & 1.57 & 1.44 & 1.62 & 1.72 & 1.50 & 1.43 & 1.41 & 1.31 & 1.25 & 1.15 & 1.26 \\
\hline 8 & 30 & & 1.29 & 1.58 & 1.67 & 1.74 & 1.62 & 1.76 & 1.70 & 1.95 & 1.52 & 1.51 & 1.22 & 1.26 & 1.38 & 1.23 & 1.24 \\
\hline 9 & 28 & 1.28 & 1.29 & 1.42 & 1.39 & 1.60 & 1.66 & 1.57 & 1.54 & 1.55 & 1.34 & 1.30 & 1.26 & 1.38 & 1.17 & 1.28 & 1.25 \\
\hline
\end{tabular}

trial of last error in all groups but group 1 (group 0 has no errors). Except for group 9, this increase has a magnitude of at least .1 sec. Mean error latencies and mean success latencies show similar results when analysed in this manner. Also, analysis of the results of Session 2 indicates that they closely resemble the results of Session 1.

The above analysis can be presented in summary form by plotting curves analogous to backward learning curves. These curves are computed by arranging all 21 groups in the manner of Table 1 and finding, for each $j,\left(\sum_{i=0}^{20} a_{i j} t_{i j} / \sum_{i=0}^{20} a_{i j}\right)$ where $t_{i j}$ is the entry in row $i$ and column $j$ and $a_{i j}$ is the number of protocols on which $t_{i j}$ is based. The result is shown in Fig. 2. There is no evidence of any consistent difference between error latency and success latency prior to the last error. However the latency on the trial of last error is considerably larger than either preceding error latencies or preceding success latencies. It is also considerably larger than any latencies after the trial of last error. It is possible that this jump in the backward curves on the trial of last error could be due to the fortuitous occurrence of a few extreme values. However, on the basis of chance, one would expect that

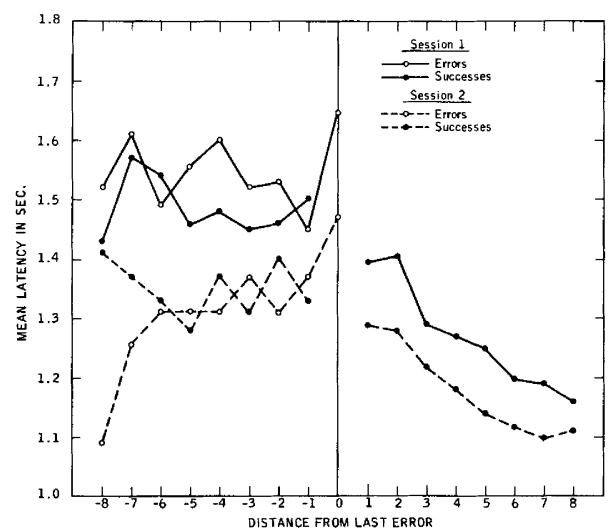

Fig. 2. Backward latency curves conditionalized on errors and successes. the last-error latency would be greater than the latency on the preceding trial in half the protocols, that it would be greater than the latency on the trial immediately after the last error in $1 / 2$ the protocols, and that it would be greater than the latencies on both the preceding trial and the next trial in $1 / 3$ of the protocols. Analysis of 383 protocols from Session 1 with last error on trial 2 or after revealed the corresponding empirical values to be $.58, .64$, and .46 , respectively. A test based on the exact binomial distribution reveals these values to be significantly greater than chance at the .001 level. Similar results were obtained with Session 2.

\section{Diseuselon}

Millward (1964) finds no evidence of a difference between error latencies and success latencies on the mean latency curve and fails to find any evidence of an increase in latency on the trial of last error. The disagreement between Millward's findings and those of the present experiment may be due to differences in experimental procedure. It may also be due to the fact that Millward does not use a learning criterion in his analysis. In the present experiment, it was found that latencies on post-criterion errors tended to be clearly lower than latencies on pre-criterion errors.

W. Kintsch has provided us with an analysis of some unpublished data obtained in connection with a 2-response, 12-item pairedassociate experiment that used nonsense syllables as stimuli (Kintsch, 1964). A table similar to Table 1 shows an increase in latency on the trial of last error for all groups except those with last error on trial 1 , although its magnitude is not large. Curiously enough, the increase does not appear on the backward latency curve. This may be due to including latencies from the group with last error on trial 1 in the averaging process. In both Kintsch's experiment and the present one, last errors that occur on trial 1 have a low average latency, being no greater than the mean latency on subsequent trials.

\section{References}

HILGARD, E. R. Methods and procedures in the study of learning. In S. S. Stevens (Ed.), Handbook of experimental psychology. New York: Wiley, 1951.

KINTSCH, W. Habituation of the GSR component of the orienting reflex during paired-associate learning. Paper read at Midwest Psychol. Ass., May, 1964.

MILLWARD, R. Latency in a modified paired-associate learning experiment. J. verbal Learn. verbal Behav., 1964, 3, 309-316. Note

1. This research has been supported by the Air Force Office of Scientific Research under Contract AF49(638)-1253 and Grant AFOSR 62-384 and by the U.S. Office of Education under Contract OE 3-10-009. 